\title{
Patrones de prescripción de psicofármacos en pacientes con esquizofrenia y trastornos relacionados internados en el Hospital Víctor Larco Herrera, 2015
}

\section{Patterns of prescription of psychotropic drugs on inpatients with schizophrenia and related disorders at Víctor Larco Herrera Hospital 2015}

\author{
Enrique Bojórquez Giraldo ${ }^{1}$, Adrián Arévalo Alván², Kelly Castro Cisneros ${ }^{2}$, Liesel Ludowieg \\ Casinelli ${ }^{2}$, Sandra Orihuela Fernández ${ }^{2}$ \\ 'Médico Psiquiatra; Doctor en Medicina; Profesor Principal, Facultad de Medicina UNMSM; Jefe de la Oficina de Docencia e Investigación Hospital Victor Larco Herrera. \\ ${ }^{2}$ Médico Residente Hospital Victor Larco Herrera.
}

\section{Correspondencia}

Enrique Bojórquez G.

enriquejavierb@outlook.es

Recibido: 11 de julio 2017.

Aceptado: 17 de agosto 2017.

Conflictos de interés: ningún

conflicto de intereses.

Financiamiento: autofinanciado.

Citar como: Bojórquez Giraldo E, Arévalo Alván A, Castro Cisneros K, Ludowieg Casinelli L, Orihuela Fernández S. Patrones de prescripción de psicofármacos en pacientes con esquizofrenia y trastornos relacionados internados en el Hospital Victor Larco Herrera, 2015. An Fac med. 2017;78(4):38692

DOI: $h$ ttp://dx.doi.org/10.15381/ anales.v78i4.14258
An Fac med. 2017;78(4):386-92 / http://dx.doi.org/10.15381/anales.v78i4.14258

\section{Resumen}

Introducción. Los antipsicóticos son la base de la terapéutica en la esquizofrenia; y en su prescripción debe evaluarse sus beneficios y riesgos potenciales. Objetivo. Identificar patrones de prescripción de psicotrópicos en pacientes hospitalizados con diagnóstico de esquizofrenia y trastornos relacionados. Diseño. Observacional, descriptivo, longitudinal, retrospectivo. Lugar. Hospital Victor Larco Herrera. Participantes. Pacientes con diagnóstico de la categoría F2 (CIE-10); mayores de 18 años y con un tiempo de estancia mínimo de 15 dias, ingresados el 2015. Intervenciones. Se evaluó las historias clínicas y se recolectaron los datos en una ficha, que posteriormente fueron vertidos en una base de datos de Microsoft Excel 2010 y analizados. Principal medida de resultados. Patrones de prescripción. Resultados. Se revisaron 174 historias clínicas. La prescripción fue agrupada en tres etapas: Inicio del tratamiento (primeros 14 días), curso del tratamiento ( 15 dias en adelante) y alta hospitalaria. El antipsicótico más usado fue la risperidona. El cambio de antipsicóticos fue mayor al inicio $(59,2 \%)$. El uso simultáneo de antipsicóticos fue $57 \%$ al inicio, reduciéndose posteriormente. La combinación más frecuente fue de antipsicóticos orales y depósito (25$40 \%)$. Otros psicofármacos usados fueron benzodiacepinas (60-80\%), estabilizadores del ánimo (50\%), y anticolinérgicos (50\%). El número promedio de medicamentos prescritos durante la hospitalización fue 4,7. Conclusiones. El patrón de prescripción encontrado fue de polifarmacia, asociado al uso simultaneo de antipsicóticos y/o medicación coadyuvante.

Palabras clave: Esquizofrenia; Psicotrópicos; Antipsicóticos; Hospitalización; Terapéutica

\section{Abstract}

Introduction. Antipsychotics are the base of therapeutic in schizophrenia; in its prescription potential benefits and risks should be assessed. Objective. Identify patterns of prescription of psychoactive drugs in patients diagnosed with schizophrenia and related disorders. Design. Observational, descriptive, Iongitudinal, retrospective. Setting. Victor Larco Herrera Hospital. Participants. Patients with diagnosis of F2 category (ICD-10); older than18 years of age, with a minimum stay of 15 days and admitted in 2015. Interventions. The medical records were evaluated and the data was collected in a datasheet, which were later poured into a Microsoft Excel 2010 database and analyzed. Main outcome measures. Patterns of prescription. Results. We reviewed 174 medical records. The most frequent diagnosis was schizophrenia (72.4\%). The prescription was grouped into three stages: Start of treatment (first 14 days), course of treatment (15 days onwards) and hospital discharge. The most commonly used antipsychotic was risperidone. The change in antipsychotics was greater at the start of treatment (59.2\%). Simultaneous use of antipsychotics was $57 \%$ at start of the treatment and subsequently reduced. The most frequent combination was oral antipsychotics and depot (25-40\%). Other psychotropic drugs used were benzodiazepines (60-80\%), mood stabilizers $(50 \%)$, and anticholinergics (50\%). The average number of medications prescribed during hospitalization was 4.74 . Conclusions. The prescription pattern found was polypharmacy, associated with the simultaneous use of antipsychotics and / or adjuvant medication.

Keywords: Schizophrenia; Psychotropic Drugs; Antipsychotics; Hospitalization; Therapeutic 


\section{INTRODUCCIÓN}

La esquizofrenia es una entidad psiquiátrica que produce alteraciones de la percepción, pensamiento, afecto y comportamiento del individuo ${ }^{(1,2)}$. Las teorías más aceptadas para explicar la esquizofrenia postulan que existe en el cerebro un aumento excesivo de los niveles de dopamina, fundamentalmente en el sistema límbico; al mismo tiempo que existe un déficit de dopamina en el lóbulo frontal, el cual afectaría negativamente el correcto funcionamiento de los procesos cognitivos y volitivos ${ }^{(3)}$.

Según la Organización Mundial de la Salud este trastorno mental tiene una incidencia de 16 a 40 por 100,000 habitantes y una prevalencia de 456 por 100,000 habitantes. Las estimaciones del riesgo en la vida de desarrollar esquizofrenia, varían entre 0,3 y $2,0 \%$, con un promedio de $0,7 \%{ }^{(4)}$.

El avance de la psicofarmacología en las últimas décadas, ha provisto a la psiquiatría de numerosas y cada vez mejores herramientas terapéuticas para el manejo de la esquizofrenia y los trastornos relacionados. Estos nuevos fármacos han mostrado beneficios indiscutibles, pero también riesgos, exigiendo racionalidad en las pautas de prescripción, de tal modo que estas se basen en las mejores evidencias científicas actuales, en una adecuada evaluación del riesgo-beneficio y en el marco ético de la relación médicopaciente.

Los antipsicóticos constituyen la base del tratamiento de la esquizofrenia y se trata de un grupo de medicamentos de naturaleza química muy heterogénea, con un mecanismo de acción común, pero no único, el bloqueo de receptores de dopamina (D2). Los antipsicóticos, en general, se dividen en dos grandes grupos: los típicos o de primera generación; y los atípicos o de segunda generación.

En el caso de los antipsicóticos típicos, todos comparten la propiedad común de bloquear los receptores dopaminérgicos (D2) ${ }^{(5)}$, difiriendo entre sí por su capacidad para bloquear los receptores de histamina, los alfa 1 adrenérgicos y los muscarínicos/colinérgicos. Sin embargo, están desprovistos de la capacidad para bloquear los receptores serotoninérgicos (5HT2).

Los antipsicóticos atípicos aumentan los niveles de dopamina en la corteza prefrontal, actuando sobre los receptores serotoninérgicos (5-HT2A), además de ser antagonistas de los receptores dopaminérgicos (D2), aunque en menor grado que los antipsicóticos típicos ${ }^{(6)}$ Uno de los desafíos actuales es elegir el fármaco más adecuado para cada paciente, lo que se hace difícil debido a la poca evidencia sobre eficacia/seguridad que ayude a elegir el antipsicótico óptimo para un paciente esquizofrénico en particular, quedando esta elección a criterio del médico tratante ${ }^{(7)}$.

Esto nos lleva a recalcar que la toma de decisiones sobre el uso de antipsicóticos parece estar condicionada por tres factores principales: la similitud de los efectos terapéuticos de todos los antipsicóticos sobre los síntomas positivos, que son los que habitualmente conducen al inicio de un tratamiento; los diferentes perfiles de efectos adversos que pueden producir, a los que se atribuye ser la causa principal de la falta de adherencia al tratamiento; y las preferencias del paciente hacia un fármaco o presentación determinados ${ }^{(8)}$.

Todo ello confirma la necesidad de individualizar el tratamiento antipsicótico, siguiendo un conjunto de recomendaciones sobre las intervenciones farmacológicas contenidas en las principales guías clínicas internacionales y las opiniones de expertos:

- El tratamiento antipsicótico debe ser integral, y por lo tanto incluir intervenciones psicosociales (psicoeducación, psicoterapia, rehabilitación psicosocial, terapia familiar, etc), además del uso de psicofármacos.

- Para el tratamiento de la fase aguda de la esquizofrenia en adultos, el médico debe escoger el fármaco según el perfil de efectos adversos, la condición clínica del paciente, el antecedente de respuesta previa a antipsicóticos y las preferencias del paciente ${ }^{(9)}$. Esto debe llevarse a cabo, en la medida de lo posible, de manera no coercitiva en combinación con intervenciones psi- cosociales que incluyan estrategias de promoción de la adherencia ${ }^{(8)}$.

- Se debe tener en consideración que los tratamientos farmacológicos deberían ser introducidos de forma gradual, a dosis terapéutica efectiva mínima, prefiriendo el uso de medicación de segunda generación, puesto que tienen una mejor tolerancia y un menor riesgo de discinesia tardía. Sin embargo, debería reconsiderarse una medicación alternativa cuando la relación riesgo-beneficio pueda cambiar para algunos pacientes (por ejemplo aumento de peso, alteración de la tolerancia a la glucosa o efectos adversos sexuales). Así también, es necesario considerar antes de cambiar un antipsicótico que el tiempo de respuesta es de cuatro a seis semanas en las dosis máximas toleradas ${ }^{(9)}$ y que solo está justificada la administración de múltiples medicamentos antipsicóticos durante los períodos de cambio de medicación ${ }^{(10)}$.

- Cabe resaltar que no se recomienda la combinación de un antipsicótico con otro, pues no está respaldada por la evidencia científica disponible; y además podría incrementar el riesgo de efectos adversos e interacciones farmacocinéticas ${ }^{(10,11)}$.

- Con respecto a las situaciones de emergencia, se debe evitar como primera elección el uso de fármacos propensos a socavar la futura adherencia al tratamiento por la producción de efectos indeseados que generen un efecto subjetivo aversivo. Los fármacos de primera generación deberían ser utilizados sólo como última opción en estas circunstancias, particularmente el haloperidol, dado que produce rigidez más que sedación. Así también, cuando es necesario el tratamiento parenteral, la vía intramuscular es preferible a la intravenosa, desde el punto de vista de la seguridad $^{(10)}$.

- En relación a la medicación de depósito, esta se debe usar únicamente en dos grupos, en aquellos que optan clara y voluntariamente por esta vía de administración; y en aquellos que, a 
pesar de una serie de intervenciones psicosociales integrales destinadas a promover la adaptación y la adherencia, repetidamente fracasan en adherirse a la medicación necesaria y presentan recaídas frecuentes, siendo aún más urgente cuando las consecuencias de las recaídas son graves e implican un riesgo sustancial tanto para el paciente como para otros. Los medicamentos inyectables de segunda generación son preferibles debido a su mejor tolerabilidad ${ }^{(10)}$.

- Con respecto al uso de fármacos no antipsicóticos como coadyuvantes tenemos que las benzodiacepinas pueden tener utilidad en fase aguda, en la agitación psicótica, pudiéndose reducir las dosis de antipsicóticos, sin embargo es necesario controlar el posible abuso de estos fármacos, y tener en cuenta que la combinación de éstos con clozapina y levomepromazina puede ser peligrosa. También, tenemos a los anticonvulsivantes que pueden ser eficaces en pacientes con conducta agitada o violenta, teniendo en cuenta que no se recomienda el uso de carbamazepina con clozapina por el potencial de ambos para producir agranulocitosis. También debemos considerar que durante el periodo de estabilidad de la esquizofrenia pueden presentarse episodios depresivos mayores, siendo necesario el uso de medicación antidepresiva ${ }^{(10)}$.

- Finalmente, deben usarse pautas terapéuticas simples, dado que a menor número de fármacos, menor cantidad de efectos adversos, menor interacción farmacológica y por tanto mayor adherencia terapéutica ${ }^{(10)}$.

El objetivo principal de la presente investigación fue identificar los patrones de prescripción de psicofármacos en pacientes con diagnóstico de esquizofrenia y trastornos relacionados (categoria F2) hospitalizados en los servicios de corta estancia del Hospital Víctor Larco Herrera (Lima, Perú) durante el año 2015, estableciendo el tipo de antipsicótico utilizado, las combinaciones entre estos y otros psicofármacos, e identificando las principales variables socio-demográficas asociadas a su prescripción, con el fin de mejorar los conocimientos del manejo farmacológico de la esquizofrenia y otros trastornos relacionados, aportando al uso racional de medicamentos en el contexto de la salud pública.

\section{MÉTODOS}

Se realizó un estudio observacional, descriptivo, longitudinal y retrospectivo en el que se incluyó a todos los pacientes de la categoría F2 de la CIE-10, con diagnóstico de esquizofrenia, trastorno esquizoafectivo, trastornos psicóticos agudos y transitorios, trastorno de ideas delirantes persistentes y otros trastornos psicóticos no orgánicos, que ingresaron a los pabellones de corta estancia del Hospital Víctor Larco Herrera durante el periodo comprendido entre el 01 de enero y el 31 de diciembre del año 2015. Asimismo, fueron excluidos del estudio aquellos pacientes cuyo tiempo de hospitalización haya sido menor de 15 días y/o que tuvieran historias clínicas incompletas y/o ilegibles.

Luego de la identificación de las historias clínicas de los pacientes según los criterios mencionados, se procedió a la recolección de la información mediante la revisión de las mismas en una ficha de recolección de datos diseñada por los investigadores y revisada por expertos. Los datos fueron vertidos en una base de datos de Microsoft Excel 2010 y fueron analizados posteriormente.

La totalidad de los procedimientos del presente estudio ha preservado la inte-

Tabla 1. Características demográficas.

\begin{tabular}{lcc}
\hline Distribución por grupos de edad & De 18 a 40 años & $66,6 \%(n=116)$ \\
& De 41 a 60 años & $29,8 \%(n=52)$ \\
& Más de 60 años & $3,4 \%(n=6)$ \\
\hline Distribución por género & Femenino & $57,4 \%(n=100)$ \\
\hline Distribución por grado de instrucción & Masculino & $42,5 \%(n=74)$ \\
& Secundaria & $61,4 \%(n=107)$ \\
& Primaria & $19,5 \%(n=34)$ \\
& Superior técnico & $10.9 \%(n=19)$ \\
& Superior universitario & $7,4 \%(n=13)$ \\
& Analfabeto & $0,5 \%(n=1)$ \\
\hline
\end{tabular}

gridad y los derechos fundamentales de los pacientes sujetos a investigación, de acuerdo con los lineamientos de las buenas prácticas clínicas y de ética en investigación biomédica. Se ha garantizado la confidencialidad de los datos obtenidos.

\section{RESULTADOS}

Se revisaron 174 historias clínicas correspondientes a la totalidad de los pacientes hospitalizados en los pabellones de corta estancia del Hospital Víctor Larco Herrera durante el periodo comprendido entre el 01 de enero de 2015 y el 31 de diciembre de 2015, y que hubiesen presentado los diagnósticos de esquizofrenia y trastornos relacionados (Categoría $\mathrm{F} 2$ de la CIE 10).

En cuanto a características demográficas, en la muestra estudiada la mayoría de los pacientes se encontraban en el rango de edad comprendido entre los 18 y 40 años de edad, eran de sexo femenino; y habían terminado la educación secundaria. (Tabla 1)

Respecto a las características clínicas (Tabla 2), se encontró que un mayor porcentaje tenía un tiempo de enfermedad mayor a cinco años, un tiempo de hospitalización mayor a un mes, y no tenía hospitalizaciones previas. El diagnóstico más frecuente fue el de esquizofrenia, seguido por el trastorno esquizoafectivo. Un pequeño grupo de pacientes presentó alguna comorbilidad psiquiátrica, como el trastorno obsesivo-compulsivo y otros trastornos de ansiedad. 
Tabla 2. Características clínicas.

\begin{tabular}{|c|c|c|}
\hline \multirow[t]{3}{*}{ Distribución por tiempo de enfermedad } & Menos de 1 año & $9,7 \%(n=17)$ \\
\hline & De 1 año a menos de 5 años & $16,0 \%(n=28)$ \\
\hline & 5 años ó más & $74,1 \%(n=129)$ \\
\hline \multirow{2}{*}{$\begin{array}{l}\text { Distribución por tiempo de } \\
\text { hospitalización }\end{array}$} & Hasta 1 mes & $61,4 \%(n=107)$ \\
\hline & Más de 1 mes & $38.5 \%(n=67)$ \\
\hline \multirow{3}{*}{$\begin{array}{l}\text { Distribución por número de } \\
\text { hospitalizaciones }\end{array}$} & 1 hospitalización & $45,9 \%(n=80)$ \\
\hline & 2 a 3 hospitalizaciones & $27,0 \%(n=47)$ \\
\hline & 4 ó más hospitalizaciones & $27,0 \%(n=47)$ \\
\hline \multirow[t]{6}{*}{ Distribución por diagnóstico } & Esquizofrenia (F20) & $72,4 \%(n=126)$ \\
\hline & Trastorno esquizoafectivo (F25) & $18,9 \%(n=33)$ \\
\hline & Trastorno psicótico agudo y transitorio (F23) & $3,4 \%(n=6)$ \\
\hline & Otros trastornos psicóticos (F28) & $2,8 \%(n=5)$ \\
\hline & Trastorno esquizotípico (F21) & $1,1 \%(n=2)$ \\
\hline & Trastorno de ideas delirantes persistentes (F22) & $1,1 \%(n=2)$ \\
\hline \multirow[t]{3}{*}{ Distribución por comorbilidad } & Sin comorbilidad & $95,4 \%(n=166)$ \\
\hline & Con trastorno de ansiedad & $2,3 \%(n=4)$ \\
\hline & Con TOC & $2,3 \%(n=4)$ \\
\hline
\end{tabular}

Tabla 3. Patrones de prescripción en las primeras dos semanas de tratamiento en los servicios de corta estancia del Hospital Víctor Larco Herrera.

\begin{tabular}{|c|c|c|}
\hline \multirow{12}{*}{$\begin{array}{l}\text { Frecuencia de antipsicóticos } \\
\text { prescritos, solos o en combinación } \\
\text { con otros antipsicóticos }\end{array}$} & Risperidona & $79 \%(n=138)$ \\
\hline & Haloperidol & $28 \%(n=50)$ \\
\hline & Decanoato de flufenazina & $18 \%(n=33)$ \\
\hline & Clorpromazina & $12,6 \%(n=22)$ \\
\hline & Decanoato de haloperidol & $10,9 \%(n=19)$ \\
\hline & Olanzapina & $5 \%(n=9)$ \\
\hline & Clozapina & $4 \%(n=7)$ \\
\hline & Sulpirida & $2,8 \%(n=5)$ \\
\hline & Quetiapina & $0,5 \%(n=1)$ \\
\hline & Trifluoperazina & $0,5 \%(n=1)$ \\
\hline & Ziprasidona & $0,5 \%(n=1)$ \\
\hline & Aripiprazol & $0,5 \%(n=1)$ \\
\hline \multirow{5}{*}{$\begin{array}{l}\text { Número de cambios de prescripción } \\
\text { de antipsicóticos }\end{array}$} & Un cambio & $78,6 \%(n=81)$ \\
\hline & Dos cambios & $13,5 \%(n=14)$ \\
\hline & Tres cambios & $4,8 \%(n=5)$ \\
\hline & Cuatro cambios & $2,9 \%(n=3)$ \\
\hline & No se realizaron cambios & $40,8 \%(n=71)$ \\
\hline \multirow{4}{*}{$\begin{array}{l}\text { Frecuencia de prescripción de } \\
\text { combinaciones de antipsicóticos }\end{array}$} & Antipsicótico único & $43 \%(n=75)$ \\
\hline & Antipsicótico oral y antipsicótico de depósito & $25 \%(n=45)$ \\
\hline & Antipsicótico oral y antipsicótico parenteral & $23,5 \%(n=41)$ \\
\hline & Dos antipsicóticos orales & $7 \%(n=13)$ \\
\hline \multirow{4}{*}{$\begin{array}{l}\text { Otros psicofármacos prescritos, en } \\
\text { combinación con antipsicóticos }\end{array}$} & Benzodiacepinas & $81,6 \%(n=142)$ \\
\hline & Estabilizadores del ánimo & $52,8 \%(n=92)$ \\
\hline & Anticolinérgicos & $52,8 \%(n=92)$ \\
\hline & Antidepresivos & $8 \%(n=14)$ \\
\hline \multirow{3}{*}{$\begin{array}{l}\text { Rango de dosis de los antipsicóticos } \\
\text { prescritos }\end{array}$} & Por encima del rango terapéutico & $0,5 \%(n=1)$ \\
\hline & En rango terapéutico & $95,5 \%(n=167)$ \\
\hline & Por debajo del rango terapéutico & $5,8 \%(n=6)$ \\
\hline
\end{tabular}

Los patrones de prescripción fueron agrupados en tres momentos:

- Etapa de inicio del tratamiento: desde el ingreso a los servicios de corta estancia hasta el decimocuarto día de hospitalización.

- Etapa de mantenimiento del tratamiento: comprendido entre el decimoquinto día de hospitalización en adelante.

- Etapa del alta hospitalaria.

a) Inicio del tratamiento (Tabla 3): El antipsicótico más empleado, sea como monoterapia o en combinación con otro antipsicótico, fue risperidona; seguido por haloperidol; y el decanoato de flufenazina. Otros antipsicóticos fueron usados con menor frecuencia. Otro hallazgo fue que en la mayor parte de pacientes se realizaron uno o más cambios de antipsicóticos, y se combinaron antipsicóticos La combinación más prescrita fue la de uno o más antipsicóticos orales con un antipsicótico de depósito. Igualmente, en el 97,1 \% de los pacientes se combinaron los antipsicóticos con otros psicofármacos; siendo los más empleados las benzodiacepinas, los estabilizadores del ánimo, y los anticolinérgicos. En general los antipsicóticos fueron empleados dentro del rango terapéutico.

b) Curso del tratamiento (Tabla 4): El antipsicótico más usado en este periodo fue, también, la risperidona, sea en monoterapia o en combinación, seguido por haloperidol, y decanoato de flufenazina. En la mayoría de los casos, en esta etapa, no se realizaron cambios de antipsicótico. En la mitad de la muestra se combinaron antipsicóticos. La combinación más prescrita fue entre un antipsicótico oral y uno de depósito. Al igual que en la etapa de inicio, la .combinación de antipsicóticos con otros psicofármacos fue la regla, siendo las más frecuentes con benzodiacepinas, estabilizadores del ánimo, y anticolinérgicos. Los antipsicóticos por lo general, fueron usados dentro del rango terapéutico.

c) Indicaciones de alta (Tabla 5): Al alta el antipsicótico más empleado fue risperidona, sea en monoterapia o en combinación; seguido por decanoato de flufenazina y haloperidol. En casi la mitad 
Tabla 4. Patrones de prescripción durante el curso del tratamiento hospitalario en los servicios de corta estancia del Hospital Víctor Larco Herrera.

\begin{tabular}{|c|c|c|}
\hline \multirow{10}{*}{$\begin{array}{l}\text { Frecuencia de antipsicóticos } \\
\text { prescritos solos o en combinación } \\
\text { con otros antipsicóticos }\end{array}$} & Risperidona & $78,2 \%(n=133)$ \\
\hline & Haloperidol & $25,9 \%(n=44)$ \\
\hline & Decanoato de flufenazina & $24,7 \%(n=42)$ \\
\hline & Clozapina & $9.4 \%(n=16)$ \\
\hline & Clorpromazina & $9.4 \%(n=16)$ \\
\hline & Sulpirida & $5,9 \%(n=10)$ \\
\hline & Quetiapina & $2,9 \%(n=5)$ \\
\hline & Olanzapina & $2,4 \%(n=4)$ \\
\hline & Trifluoperazina & $1,2 \%(n=2)$ \\
\hline & Aripiprazol & $0,6 \%(n=1)$ \\
\hline \multirow{4}{*}{$\begin{array}{l}\text { Número de cambios de } \\
\text { prescripción de antipsicóticos }\end{array}$} & Un cambio & $27,6 \%(n=47)$ \\
\hline & Dos cambios & $3,5 \%(n=6)$ \\
\hline & Cuatro cambios & $0,6 \%(n=1)$ \\
\hline & No se realizaron cambios & $68,2 \%(n=116)$ \\
\hline \multirow{4}{*}{$\begin{array}{l}\text { Frecuencia de combinaciones de } \\
\text { antipsicóticos }\end{array}$} & Antipsicótico único & $50 \%(n=85)$ \\
\hline & Antipsicótico oral y antipsicótico de depósito & $27,1 \%(n=46)$ \\
\hline & Dos antipsicóticos orales y antipsicótico de depósito & $5,3 \%(n=9)$ \\
\hline & Dos antipsicóticos orales & $2,3 \%(n=4)$ \\
\hline \multirow{5}{*}{$\begin{array}{l}\text { Otros psicofármacos prescritos en } \\
\text { combinación con antipsicóticos }\end{array}$} & Benzodiacepinas & $77,1 \%(n=131)$ \\
\hline & Anticolinérgicos & $52,4 \%(n=89)$ \\
\hline & Estabilizadores del ánimo & $41,8 \%(n=71)$ \\
\hline & Antidepresivos & $6,5 \%(n=11)$ \\
\hline & Otros & $4,7 \%(n=8)$ \\
\hline \multirow{3}{*}{$\begin{array}{l}\text { Rango de dosis de los } \\
\text { antipsicóticos prescritos }\end{array}$} & Por encima del rango terapéutico & $5,2 \%(n=9)$ \\
\hline & En rango terapéutico & $94,1 \%(n=160)$ \\
\hline & Por debajo del rango terapéutico & $0,6 \%(n=1)$ \\
\hline
\end{tabular}

de la muestra se realizaron combinaciones de antipsicóticos. La combinación más prescrita fue entre un antipsicótico oral y uno de depósito. También la combinación de antipsicóticos con otros psicofármacos fue alta. La mayor combinación fue con benzodiacepinas, seguido de anticolinérgicos, y estabilizadores del ánimo. Los antipsicóticos prescritos se encontraban en el rango terapéutico.

Estos hábitos de manejo farmacológico nos indican que en la prescripción había una prevalencia de polifarmacia, encontrándose que a lo largo de toda la hospitalización, el 94,8\% de los pacientes recibieron tres o más fármacos en su tratamiento, mientras que el 55,1\% recibieron cinco o más; por lo que el promedio de psicofármacos empleados por paciente durante la hospitalización fue de 4,7 medicamentos.

Todos estos datos nos indican que el hallazgo principal de este estudio es que el patrón general de prescripción de psicofármacos en pacientes con diagnóstico de esquizofrenia y trastornos relacionados (F2) hospitalizados en los servicios de corta estancia del Hospital Víctor Larco Herrera durante el año 2015 tuvo las siguientes características:

- El antipsicótico más empleado en todo el curso de la hospitalización fue

- Durante la hospitalización el cambio de antipsicóticos fue alto. la risperidona.
- El uso simultáneo de antipsicóticos durante el tratamiento también fue alto.

- La combinación más frecuente fue la de un antipsicótico oral con un antipsicótico de depósito.

- La combinación con otros psicofármacos también fue alta, con benzodiacepinas entre $60 \%$ a $80 \%$, estabilizadores del ánimo alrededor del 50\%, y anticolinérgicos en un $50 \%$.

- El uso de antipsicóticos de depósito fue alto, generalmente incorporado desde el inicio de la hospitalización y en combinación con otros antipsicóticos.

- El uso de clozapina estuvo por debajo del $10 \%$.

- El patrón de prescripción fue de polifarmacia.

\section{DISCUSIÓN}

El presente estudio permitió determinar los patrones de prescripción de fármacos antipsicóticos utilizados con mayor frecuencia en los pacientes hospitalizados de la categoría F2, en los servicios de corta estancia del Hospital Víctor Larco Herrera.

Las características demográficas fueron similares a las de las muestras halladas en otros estudios realizados en Sudamérica ${ }^{(7,12)}$, difiriendo de estudios en otras regiones como Europa y Asia ${ }^{(13,14,15)}$. El diagnóstico establecido con mayor frecuencia durante el ingreso hospitalario fue el de esquizofrenia $(72,4 \%)$, lo que concuerda con la literatura internacional ${ }^{(12,15)}$. A diferencia de lo hallado en el estudio de Fisher y col. en la que se encontró $10,6 \%$ de trastornos de ansiedad; en este estudio se encontró $2,3 \%$ de trastornos de ansiedad y $2,3 \%$ de trastorno obsesivo-compulsivo ${ }^{(16)}$.

Los antipsicóticos más usados durante el transcurso de la hospitalización y al momento del alta fueron los atípicos, tanto en monoterapia como en combinación, lo que confirma su posición como principal opción terapéutica y es coincidente con la literatura internacio- 
Tabla 5. Patrones de prescripción al alta hospitalaria en los servicios de corta estancia del Hospital Víctor Larco Herrera.

\begin{tabular}{|c|c|c|}
\hline \multirow{8}{*}{$\begin{array}{l}\text { Frecuencia de antipsicóticos } \\
\text { prescritos al alta, solos o } \\
\text { en combinación con otros } \\
\text { antipsicóticos }\end{array}$} & Risperidona & $78,4 \%(n=131)$ \\
\hline & Decanoato de flufenazina & $30,5 \%(n=51)$ \\
\hline & Halperidol & $20,9 \%(n=35)$ \\
\hline & Clozapina & $6,5 \%(n=11)$ \\
\hline & Clorpromazina & $5,9 \%(n=10)$ \\
\hline & Sulpirida & $2,3 \%(n=4)$ \\
\hline & Olanzapina & $2,3 \%(n=4)$ \\
\hline & Quetiapina & $2,3 \%(n=4)$ \\
\hline \multirow{4}{*}{$\begin{array}{l}\text { Frecuencia de combinaciones de } \\
\text { antipsicóticos prescritos al alta }\end{array}$} & Antipsicótico único & $55 \%(n=92)$ \\
\hline & Antipsicótico oral y antipsicótico de depósito & $40,4 \%(n=67)$ \\
\hline & Dos antipsicóticos orales y antipsicótico de depósito & $2,3 \%(n=4)$ \\
\hline & Dos antipsicóticos orales & $2,3 \%(n=4)$ \\
\hline \multirow{5}{*}{$\begin{array}{l}\text { Otros psicofármacos prescritos } \\
\text { al alta hospitalaria, en } \\
\text { combinación con antipsicóticos }\end{array}$} & Benzodiacepinas & $64 \%(n=107)$ \\
\hline & Anticolinérgicos & $52 \%(n=87)$ \\
\hline & Estabilizadores del ánimo & $46,7 \%(n=78)$ \\
\hline & Antidepresivos & $7,7 \%(n=13)$ \\
\hline & Otros & $1,2 \%(\mathrm{n}=2)$ \\
\hline \multirow{3}{*}{$\begin{array}{l}\text { Rango de dosis de los } \\
\text { antipsicóticos prescritos }\end{array}$} & Por encima del rango terapéutico & $3 \%(n=5)$ \\
\hline & En rango terapéutico & $96,4 \%(n=161)$ \\
\hline & Por debajo del rango terapéutico & $0,6 \%(n=1)$ \\
\hline
\end{tabular}

nal ${ }^{(7,12,15,17)}$. De los antipsicóticos atípicos, el más frecuentemente empleado fue la risperidona, similar a lo hallado en estudios realizados en EEUU y Chile $(7,16)$; pero con la salvedad que es el único atípico incluido en el Petitorio Nacional de Medicamentos además de ser el de menor costo; y los menos empleados fueron el aripiprazol y la ziprasidona, posiblemente debido a su alto costo ${ }^{(15)}$.

Pese a las recomendaciones internacionales, que favorecen el uso de un solo antipsicótico, evitando las combinaciones, y hacen referencia a que no existe evidencia de mayor beneficio terapéutico con el uso de más de un antipsicótico (11); el uso de antipsicóticos en combinación en este estudio fue del $57 \%$ al ingreso y $45 \%$ al alta, siendo estos valores elevados, similares a los valores hallados en estudios realizados en otros países ${ }^{(12,15,16,18)}$.

Resaltan los frecuentes cambios de antipsicóticos durante el curso de la hospitalización, no respetándose las recomendaciones de las guías clínicas internacionales ${ }^{(19)}$.
Respecto al patrón de prescripción, en el estudio se evidencio que era común adicionar al antipsicótico otros psicofármacos. Así, la adición de benzodiacepinas es alta (60 a 80\%), combinación que coincide con lo hallado en un trabajo español (15); pero es alto comparado con otro trabajo hecho en Colombia, donde se halló un $26 \%{ }^{(18)}$. Sin embargo, lo que más llama la atención es el uso elevado de estabilizadores del ánimo, si lo comparamos con lo hallado en otros trabajos que tiene como principal diagnóstico a la esquizofrenia. También, destaca el uso de anticolinérgicos, que se da en la mitad de los pacientes de la muestra, incluso al alta, lo cual difiere con otros estudios ${ }^{(15,18)}$. Finalmente, cabe destacar que en el estudio estas combinaciones se dieron desde el inicio hasta el alta; con un promedio de 4,7 medicamentos por paciente; lo que indica claramente un patrón de polifarmacia.

Respecto al uso de antipsicóticos prescritos dentro de los rangos terapéuticos, consideramos que es un dato que puede resultar engañoso, si se tiene en cuenta el patrón de polifarmacia dominante en el hospital; lo cual implica múltiples interacciones, probablemente algunas peligrosas.

Otro hallazgo relevante, fue el uso elevado de antipsicóticos de depósito, que es incorporado desde el inicio de la hospitalización, manteniéndose hasta el alta y en combinación con otros antipsicóticos. Estos resultados concuerdan con algunos estudios respecto a su frecuencia de uso; pero difieren respecto al momento de la prescripción y a su alta combinación con otros antipsicóticos ${ }^{(12,18)}$.

En cuanto al uso de clozapina, los resultados muestran que su uso se da en menos del $10 \%$ de pacientes, lo cual coincide con otros estudios ${ }^{(7,12)}$. Sin embargo, si tomamos en cuenta las características de la muestra, formada en un elevado porcentaje por pacientes con más de 5 años de enfermedad y varias hospitalizaciones, su uso debería ser más extendido.

El promedio de polifarmacia hallado en el estudio fue de 4,7, lo cual llama la atención ya que si bien es cierto no hay suficientes estudios comparativos en psiquiatría respecto a este tema, la investigación actual y la práctica basada en evidencias, respalda el uso de monoterapia antipsicótica debido a que aporta un mejor equilibrio entre riesgos y beneficios en el tratamiento de primera línea de la esquizofrenia y los trastornos relacionados ${ }^{(20)}$. De las conclusiones del presente estudio se ha recomendado a la Dirección General del Hospital la programación de un curso sobre uso racional de antipsicóticos dirigido al personal médico; y la elaboración de una directiva que regule la prescripción de psicofármacos en pacientes esquizofrénicos y trastornos relacionados.

\section{REFERENCIAS BIBLIOGRÁFICAS}

1. Tandon R, Keshavan MS, Nasrallah HA. Schizophrenia, "just the facts": what we know in 2008. Part 1: Overview. Schizophr Res. 2008; 100:4-19. doi: 10.1016/j.schres.2008.01.022.

2. Sadock B, Sadock V. Sinopsis de psiquiatría. $10^{\mathrm{a}}$ edición. Baltimore: Williams and Wilkins; 2009. p. 467-97.

3. Brisch, R, Saniotis A, Wolf R, Bielau H, Bernstein $\mathrm{H}$, Steiner J, et al. The Role of Dopamine in Schi- 
zophrenia from a Neurobiological and Evolutionary Perspective: Old Fashioned, but Still in Vogue. Front Psychiatry. 2014; 5: 47. doi: 10.3389/ fpsyt.2014.00047

4. Tandon R, Keshavan MS, Nasrallah HA. Schizophrenia, "just the facts" what we know in 2008. Part 2: Epidemiology and etiology. Schizophr Res. 2008; 102:1-18. doi: 10.1016/j.schres.2008.04.011

5. Miller, R. Mechanisms of Action of Antipsychotic Drugs of Different Classes, Refractoriness to Therapeutic Effects of Classical Neuroleptics, and Individual Variation in Sensitivity to their Actions: Part I. Curr Neuropharmacol, 2009 Dec; 7(4): 302-314 doi: 10.2174/157015909790031229

6. Meltzer, H. Mechanism of Action of Atypical Antipsychotic Drugs. En: K. L. Davis, D. Charney, J. T. Coyle, C. Nemeroff. Neuropsychopharmacology: The Fifth Generation of Progress, 5th edition. Philadelphia. Lippincott, Williams, \& Wilkins, 2002. p 819-831.

7. Nieto RR., Silva H. Prescripción de antipsicóticos atípicos en pacientes hospitalizados de la Clínica Psiquiátrica Universitaria. Rev Chil Neuro-Psiquiat 2008; 46 (1): 16 - 24. http://dx.doi.org/10.4067/ S0717-92272008000100003

8. Ceruelo Bermejo J y Garcia Rodicio S Antipsicóticos típicos. Antipsicóticos atipicos FMC. 2007;14(10):637-47

9. Ministerio de Salud y Protección Social-Colciencias. Guía de práctica clinica para el diagnóstico, tratamiento e inicio de la rehabilitación psicosocial de los adultos con esquizofrenia. Sistema General de Seguridad Social en Salud-Colombia. Centro Nacional de Investigación en Evidencia y Tecnologias en Salud CINETS. Guia No 29. Bogotá, Colombia. Junio 2014.

10. Grupo de trabajo de la Guia de Práctica Clinica sobre la Esquizofrenia y el Trastorno Psicótico Incipiente. Fórum de Salut Mental, coordinación. Guía de Práctica Clínica sobre la Esquizofrenia y el Trastorno Psicótico Incipiente. Madrid: Plan de Calidad para el Sistema Nacional de Salud del Ministerio de Sanidad y Consumo. Agència d'Avaluació de Tecnologia i Recerca Mèdiques; 2009. Guia de Práctica Clínica: AATRM. No 2006/05-2.

11. Galling B., Roldán A., Hagi K., Rietschel L., Walyzada F., Zheng W, et al. Antipsychotic augmentation vs. monotherapy in schizophrenia: systematic review, meta-analysis and meta-regression analysis. World Psychiatry. 2017;16:77-89 DOI:10.1002/ wps. 20387

12. Toledo M, Mato M, Olmos I, Frontini MA, Porteiro $F$, Labraga $P$, et al. Prescripción de antipsicóticos al egreso hospitalario durante el periodo abrilsetiembre de 2012 en el Hospital Vilardebó de Montevideo, Uruguay. Rev Psiquiatr Urug. 2015; 79(1): $29-38$.

13. Chong MY, Tan $\mathrm{CH}$, Fujii S, Yang SY, Ungvari GS, Si T, et al. Antipsychotic drug prescription for schizophrenia in East Asia: rationale for change. Psychiatry and Clinical Neuroscience. 2004, 58, 61-67.

14. Sarkar P, Chakraborty K, Misra A, Shukla R, Swain SP. Pattern of psychotropic prescription in a tertiary care center: A critical analysis. Indian Journal of Pharmacology. 2013, 45, 3: 270 - 273. DOI: 10.4103/0253-7613.111947

15. Montejo AL. Majadas S., Mayoral F., Sanjuán J., Ros S., Olivares JM., et al. Análisis de los patrones de prescripción de antipsicóticos en psiquiatria. Actas Esp Psiquiatr. 2006;34(5):323-329.

16. Fisher M, Reilly K, Isenberg K, Villa K.F. Antipsychotic patterns of use in patients with schizophrenia: polypharmacy versus monotherapy. BMC Psychiatry. 2014; 14:341. doi: 10.1186/s12888-014-0341-5.

17. Mundt AP, Aichberger MC, Fakhriddinov $S$, Fayzirahmanova M, Grohmann R, Heinz A, et al. Prescription patterns of patients diagnosed with schizophrenia in mental hospitals in Tashkent/Uzbekistan and in four German cities. Pharmacoepidemiol Drug Saf. 2012; 21:145-151. DOI: 10.1002/ pds. 2166

18. Machado-Alva JE, Morales-Plaza CD. Patrones de prescripción de antipsicóticos en pacientes afiliados al Sistema General de Seguridad Social en Salud de Colombia.Biomédica,2013;33:418-28 Doi:http://dx.doi.org/10.7705/ biomedica.v33i3.1529

19. Taylor D, Paton C, Kapur S. The Maudsley Prescribing Guidelines in Psychiatry. 12th edition. United Kingdom: Wiley Blackwell; 2015. p. 20.

20. Stahl, S. Nuevas guias para el uso de la polifarmacia antipsicótica. Rev Paiquiatr Salud Ment. 2013;6(3):97-100. DOI: 10.1016/j. rpsm.2013.01.001 